\title{
Measuring Method for Diameter of Bearings Based on the Edge Detection Using Zernike Moment
}

\author{
Zhang Weipeng* \\ College of Electrical and Mechanical Engineering, Ningbo Dahongying University, Ningbo, 315000 China
}

\begin{abstract}
In addressing the application problem of digital image technology in the high-precision measurement of the diameter of bearings, a measuring method for diameter of bearings based on the edge detection using Zernike moments is proposed. First, the images of bearings are subject to bilateral filtering based on wavelet transformation, and the images after pretreatment are subject to the initial setting of pixel level by using Laplacian of Gaussian operator. Then edges are positioned accurately by using the subpixel Zernike moments. The result shows that the positioning precision of edges is high with this method. A subpixel nondestructive detection of the external diameter of bearing is realized, and the detection precision reaches the standards of industrial measurement.
\end{abstract}

Keywords: Diameter of bearings, Subpixel, Zernike moments, Edge detection.

\section{INTRODUCTION}

Bearing is a very important and widely used rotating component in the machinery industry with large production volume and high requirement for accuracy. Currently, many bearing manufacturers in China still depend on mechanical and optical measuring instruments to inspect the accuracy of the bearing size, and these processes are relatively backward. The efficiency and accuracy of inspection method are usually low, and the method is vulnerable to human-related errors. Machine vision is a noncontact measuring technique based on image processing technology with accurate and reliable detection results. Moreover, among the traditional edge detection algorithms such as Sobel operator and Robert operator [1-2], the Laplacian of Gaussian operator is an effective detection method. However, the positioning precision is only of the integer pixel level, which can no longer satisfy the requirement for high-precision measurement of bearings. Subpixel means that each pixel in the image is further divided into smaller units to achieve higher precision. The subpixel detection method has been widely used for the highprecision measurements [3].

So far, the subpixel edge detection algorithms mainly include the interpolation method, fitting method and moment method. Related researches [4-5] show that interpolation method has a small calculation amount, short computation time, good repeatability and strong anti-disturbance ability, but the positioning precision is low. The fitting method has strong anti-disturbance ability and high positioning precision. But there are contradictions between the antidisturbance ability and detection of edges in complex shape. The moment method has high positioning precision, but the calculation amount is large and the computation time is long.

*Address correspondence to this author at the college Road, Ningbo, China; Postcard: 315000; Tel: 13685720826; E-mail: zwphb@163.com
The edge detection performed in this study is to process the images of rolling bearings. The shapes of the edge are simple. Considering the advantages and disadvantages of the introduced methods and the actual situation of measurement, the subpixel edge detection algorithm based on Zernike moments is chosen. Its positioning precision and computation time are better than those of other space operators.

\section{PRETREATMENT OF IMAGES}

CMOS sensor and amplification factor of 400 was used. The USB interface was used to collect data. The original collected data is shown in Fig. (1a).

Images are unavoidably affected by random disturbance and noise during collection, so they need to be pretreated. The image denoising methods are mainly in space domain and frequency domain [6-7]. Both of them are essentially $a$ bandpass filter using the isotropic filtering method. The defect of such methods is that the edge information of the images will be obscured and even destroyed during denoising. Bilateral filters can keep the image edges smooth. However, the images after filtering will still become obscured [8-9].

In this paper, bilateral filtering is performed based on wavelet decomposition. First, the original images are subject to the one-level wavelet decomposition. Signals are decomposed into high frequency components and low frequency components through wavelet decomposition. Then the high frequency subband images, $\mathrm{H}, \mathrm{V}$ and $\mathrm{D}$, are subject to bilateral filtering. The source images are recovered by using the invert wavelet transform. The obtained recovered images are balanced in terms of denoising and enhancement of detail features with the image enhancement algorithm. Intact edge information of the images is obtained, and the images after pretreatment are shown in Fig. (1b). So far intact edge information can be obtained by edge extraction from the preprocessing results. 


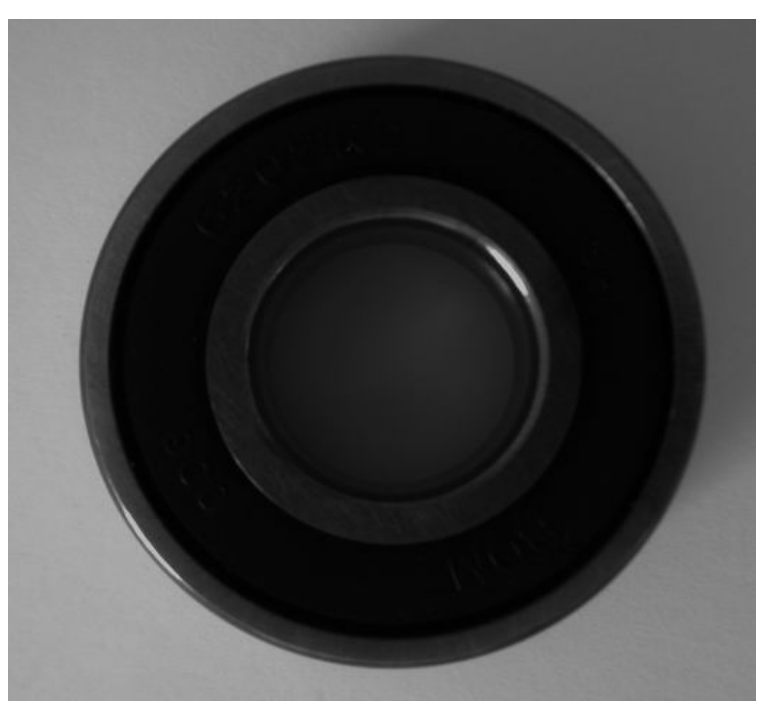

(a) The original images

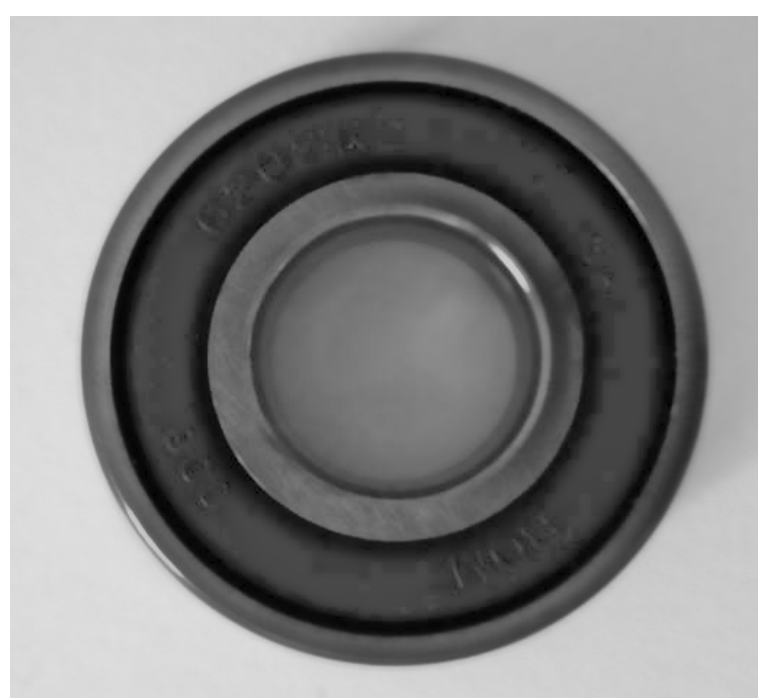

(b) The image of bilateral filtering based on wavelet transformation;

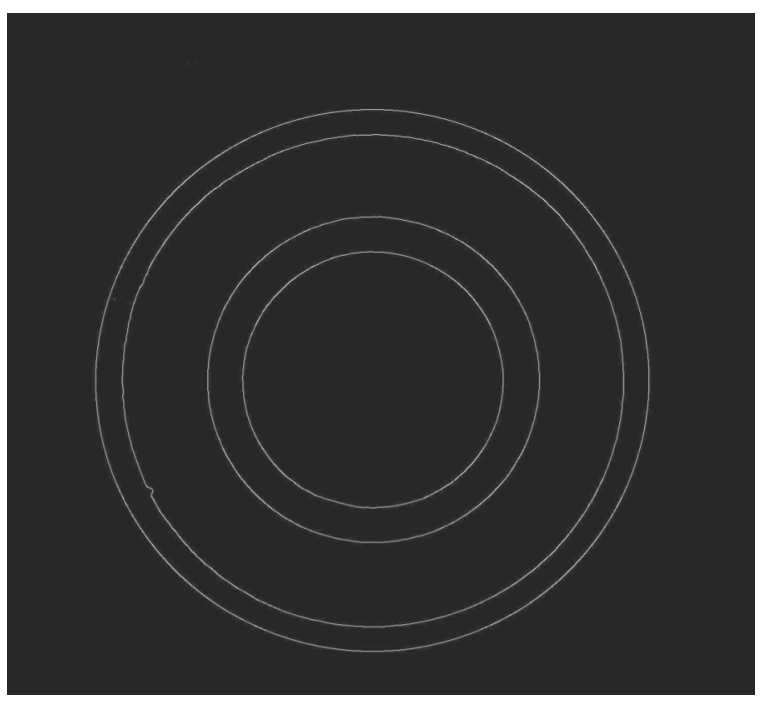

(c) The image of edge extraction

Fig. (1). Experimental Images. 


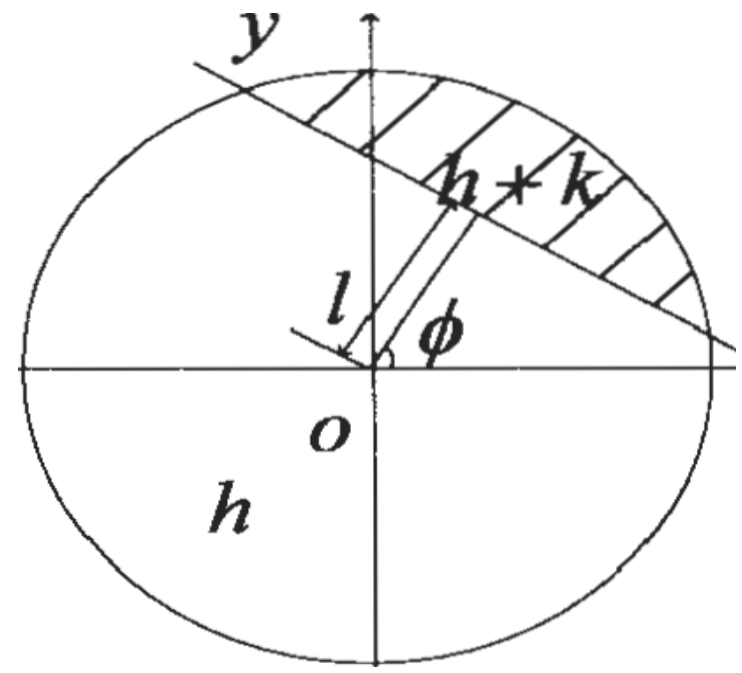

Fig. (2). Ideal Two-Dimensional Boundary Model.

\section{EDGE POSITIONING}

\subsection{Edge Positioning of Pixel Levels}

The goal of pixel level edge positioning is to preliminarily locate the target edge by using the single-pixel edge detection algorithm. Thus, the positioning precision of a single pixel can be obtained. Common pixel-level edge detection algorithms include Sobel operator, Prewitt operator, Laplacian operator and Laplacian of Gaussian operator. Sobel operator and Prewitt operator can provide intact and accurate edge information. But many pseudo edges will be detected at the same time, hence the edge positioning precision is low. In actual image processing, Laplacian operator is not generally used directly since it is very sensitive to noise. The Laplacian image processing will generate the width of bilateral edges [10]. In this paper, the Laplacian of Gaussian operator is used as the edge positioning function of integer pixel level as it has the functions of filtering noise and detecting edges of the images.

Laplacian of Gaussian is an algorithm that combines the Gaussian filter and Laplacian edge detection. When the Laplacian of Gaussian operator is used to extract the edges, the Gaussian smoothing template and original images are subject to convolution operation to achieve the goal of denoising. Then the Laplacian operator of the processed image is computed. Finally the point with the Laplacian value of 0 in the detected image is selected as the edge point. The experiment shows [11] that the method can achieve the denoising of the infrared images, and detailed information of edge of the images can also be preserved. The effect of edge detection is usually satisfactory.

Two-dimensional Gaussian smoothing function is defined as follows.

$G(x, y)=\frac{1}{2 \pi \sigma^{2}} e^{-\frac{x^{2}+y^{2}}{2 \sigma^{2}}}$

where $x, y$ is the image coordinate, and $\sigma$ is the standard deviation of Gaussian distribution.
The original image is supposed as $f(x, y)$. The output image $h(x, y)$ is obtained through convolution operation using Laplace operator.

$h(x, y)=\nabla^{2}(g(x, y) \otimes f(x, y))$

where $\otimes$ is the convolution, and Laplacian operator is

$\nabla^{2} G(x, y)=\frac{\partial^{2} G}{\partial x^{2}}+\frac{\partial^{2} G}{\partial y^{2}}$.

The Laplacian value is calculated from the image after using Gaussian smoothing to obtain the Laplacian of Gaussian operator $\nabla^{2} h(x, y)$. The expression is shown as follows.

$\nabla^{2} h(x, y)=\frac{1}{\pi \sigma^{4}}\left(\frac{x^{2}+y^{2}}{2 \sigma^{2}}-1\right) e^{-\frac{x^{2}+y^{2}}{2 \sigma^{2}}}$

The edge [12] of image $f(x, y)$ can be obtained by computing the track by which $\nabla^{2} h(x, y)$ passes through the zero point. Then the edge image is extracted after scanning the whole image for once. The precision of image edge at this time is only accurate to the pixel level. The image after edge processing is shown as Fig. (1c).

\subsection{Edge Positioning Using Subpixel Zernike Mo- ments}

The basic idea of edge detection with Zernike moment is to simulate the actual edges by using idealized edge model. Four edge parameters are obtained by computing 3 Zernike moments with different orders of images. These parameters are used to determine the subpixel coordinates [13] of the edges. The established ideal step edge model [14-16] is shown in Fig. (2). The part of straight line of unit circle contained in the circle represents the ideal edge. Gray values of both sides of edge in the circle are $h$ and $h+k$, respectively. 


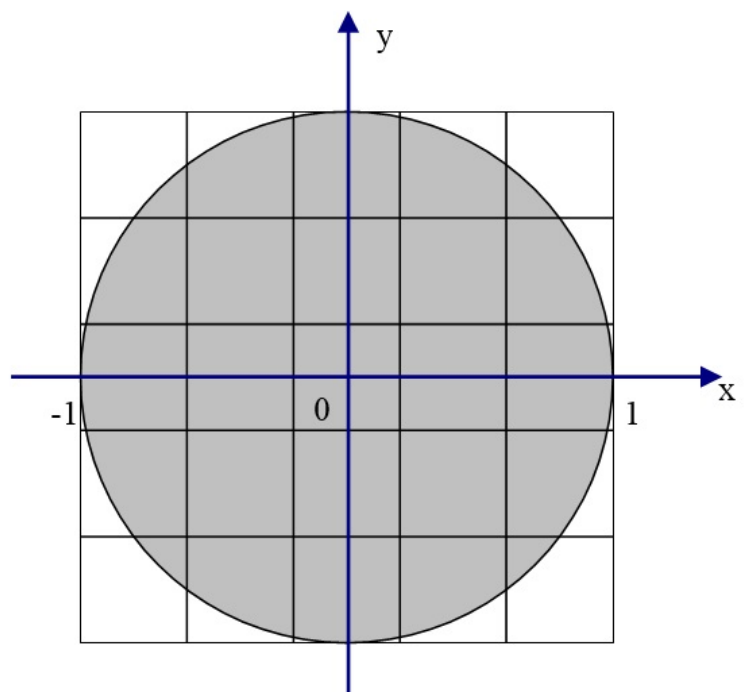

Fig. (3). Unit Circle Defined in the $5 \times 5$ Pixel Region.

$k$ is the difference between the gray values of both sides of the edge. $l$ is the vertical distance from the center of unit circle to the edge. $\phi$ is the included angle between the edge and $x$ axis.

The original image is $f(x, y)$. The $n$-order $m$-degree Zernike moment of the image are defined as follows.

$$
A_{n m}=\frac{n+1}{\pi} \iint_{x^{2}+y^{2} \leq 1} f(x, y) V_{n m}^{*}(\rho, \theta) d x d y
$$

Where, $V_{n m}(\rho, \theta)$ is the orthogonal $n$-order $m$-degree Zernike moment polynomial in the unit circle of polar coordinate system. $*$ represents the complex conjugate.

Zernike moment has rotation invariance [17]. That is, the modules of Zernike moment before rotation $A_{n m}$ and after rotation $A_{n m}^{\prime}$ remain the same and only the phase changes. The relationship can be expressed as follows.

$$
A_{n m}=A_{n m}^{\prime} e^{-j m \phi}
$$

The edge is made parallel with $y$ axis after clockwise rotation of $\phi$. Then there is the following formula.

$$
\iint_{x^{2}+y^{2} \leq 1} f^{\prime}(x, y) y d y d x=0
$$

Where, $f^{\prime}(x, y)$ is the image after rotation.

According to formulas (5) and (6), Zernike complex polynomial can be obtained:

$V_{00}=1, V_{11}=x+j y, V_{20}=2 x^{2}+2 y^{2}-1$
The relationship between the Zernike moment of image before rotation and after rotation is shown as the following formula.

$$
A_{\mathrm{oO}}^{\prime}=A_{00}, A_{11}^{\prime}=A_{11} e^{j \varphi}, A_{20}^{\prime}=A_{20}
$$

In this paper, the diameter of planar circle of image is taken as 5 pixels. The sampling window is taken as $5 \times 5$ pixels. The unit circle is inscribed within the square, and divided into an average of $5 \times 5$ grid, as shown in Fig. (3). The related parameters of Zernike moment are calculated:

Three matrices are used. They are $A_{00}, A_{11}$ and $A_{20}$, respectively. Hence in the shaded area in each grid, $V_{00}, V_{11}$ and $V_{20}$ are integrated, respectively. The integral templates of the corresponding pixels can be obtained.

The subpixel edge detection using Zernike moment uses the following equation:

$\left[\begin{array}{l}x_{s} \\ y_{s}\end{array}\right]=\left[\begin{array}{l}x \\ y\end{array}\right]+\frac{N l}{2}\left[\begin{array}{c}\cos \phi \\ \sin \phi\end{array}\right]$

Where, $\left(x_{s}, y_{s}\right)$ is the subpixel coordinate. $(x, y)$ is the edge in rough positioning, and $l$ is the straight-line distance between the origin of coordinates and the edge.

$\left(x_{1}, y_{1}\right)$ and $\left(x_{2}, y_{2}\right)$ are the two coordinates of circles in the image determined by using the subpixel positioning method. $d$ is the external diameter of the measured bearing. See formula (10).

$$
d^{2}=\left(x_{1}-x_{2}\right)^{2}+\left(y_{1}-y_{2}\right)^{2}
$$


Table 1. Measured Values of Subpixel External Diameter of Bearings.

\begin{tabular}{|c|c|c|c|c|}
\hline $\begin{array}{c}\text { the mmber of } \\
\text { measure- } \\
\text { ments }\end{array}$ & pixel & $\begin{array}{c}\text { external diameter } \\
\text { of } \\
\text { bearings } / \mathrm{mm}\end{array}$ & $\begin{array}{c}\text { the measure- } \\
\text { ment } \\
\text { deriation } / \mathrm{mm}\end{array}$ & $\begin{array}{c}\text { the } \\
\text { relative } \\
\text { error(\%) }\end{array}$ \\
\hline 1 & 540.119 & 25.010397 & 0.008397 & 0.003 \\
\hline 2 & 540.137 & 25.011184 & 0.009184 & 0.037 \\
\hline 3 & 539.670 & 24.989599 & -0.012440 & -0.050 \\
\hline 4 & 540.305 & 25.018960 & 0.016963 & 0.068 \\
\hline 5 & 538.943 & 24.953071 & -0.00489 & -0.196 \\
\hline 6 & 539.882 & 24.955890 & -0.046412 & -0.186 \\
\hline 7 & 541.012 & 25.04886 & 0.046856 & 0.187 \\
\hline 8 & 540.897 & 25.043531 & 0.041531 & 0.166 \\
\hline 9 & 539.798 & 24.992647 & -0.009353 & -0.04 \\
\hline 10 & 540.256 & 25.013853 & 0.011853 & 0.05 \\
\hline
\end{tabular}

Table 2. Measured Values of Subpixel Inner Diameter of Bearings.

\begin{tabular}{|c|c|c|c|c|}
\hline $\begin{array}{c}\text { the mmber of } \\
\text { measure- } \\
\text { ments }\end{array}$ & pixel & $\begin{array}{c}\text { inner } \\
\text { diameter of } \\
\text { bearings } / \mathrm{mm}\end{array}$ & $\begin{array}{c}\text { the measure } \\
\text { ment } \\
\text { deviation } / \mathrm{mm}\end{array}$ & $\begin{array}{c}\text { the } \\
\text { relative } \\
\text { error(\%) }\end{array}$ \\
\hline 1 & 324.005 & 15.003136 & 0.001136 & 0.008 \\
\hline 2 & 323.853 & 14.996104 & -0.00890 & -0.059 \\
\hline 3 & 324.102 & 15.007633 & 0.002634 & 0.018 \\
\hline 4 & 323.896 & 14.998095 & -0.0031905 & -0.021 \\
\hline 5 & 324.310 & 15.0172653 & 0.0152653 & 0.101 \\
\hline 6 & 324.544 & 15.026387 & 0.021387 & 0.142 \\
\hline 7 & 324.736 & 15.035277 & 0.030277 & 0.202 \\
\hline 8 & 323.987 & 15.000598 & -0.004402 & -0.030 \\
\hline 9 & 324.341 & 15.016988 & 0.011988 & 0.080 \\
\hline 10 & 324.880 & 15.041944 & 0.036944 & 0.246 \\
\hline
\end{tabular}

Pixel diameter $d$ multiplied by the resolution of image surface is the actual size of bearing.

\section{RESULT ANALYSIS}

The resolution of the current image surface is calculated as $0.04630526 \mathrm{~mm} / \mathrm{pixel}$ by using the standard component of $1 \mathrm{~cm} \times 1 \mathrm{~cm}$ chessboard. The system is used to measure the standard bearing. The actual values of external diameter and internal diameter of bearing are $25.002 \mathrm{~mm}$ and $15.005 \mathrm{~mm}$, respectively. Ten groups of data are obtained as shown in Table $\mathbf{1}$ and Table $\mathbf{2}$.

Accurate positioning of image edge is an important factor influencing the measuring precision of the image. The antinoise ability of the algorithm should be also considered. The critical issue to realize for the edge detection is to establish an edge model that accords with reality to describe the status of characteristic edges of the image. It can be seen from the measuring results that the average external diameter of this group is $25.004077 \mathrm{~mm}$ and the average internal diameter is $15.014343 \mathrm{~mm}$. In comparison with the theoretical values, the measurement deviation is very small, and the measuring precision error is less than $3 \%$. The measuring method for the diameter of bearings introduced in this paper can achieve a high measuring precision and the noncontact and real-time measurement of the diameter of bearings can be realized.

\section{CONFLICT OF INTEREST}

The author confirms that this article content has no conflict of interest.

\section{ACKNOWLEDGEMENTS}

This work was financially supported by Public Welfare Program of Zhejiang Province (Number: 2014C31161, 2014C31160), Natural science foundation project of Ningbo City (2013A10100).

\section{REFERENCES}

[1] G. Fu, "A parallel algorithm for image edge detection based on the Sobel operator", Microelectron. Comput., vol. 23, pp. 132-134, September 2006.

[2] F. Zhao, X. Luan, and Y. Sun, "Comparison and analysis of detections with several edge detection operators for digital images", Tech. Automat. Appl., vol. 28, pp. 68-72. March 2009.

[3] F. Yu, S. Zhong, M. Xie and J. Sun, "Research for high-precision measurement of the size of small connection pieces", Appl. Electron. Tech., vol. 38, pp. 144-146, September 2012,

[4] Z. Li, Y. Liu, M. Sun, W. Wang, Y. Xia, L. Zhang and W. Zhang, "Parameter measurement system of PCR chip based on the edge detection with sub pixel", Instrum. Tech. Sensor, no. 2, pp. 65-67, 89, Feb. 2014

[5] B. Zhang, D. Zheng and C. Sun, "Measurement for weak light signals based on pseudorandom sequence", Acta Electron. Sin., pp. 212-214. September 2009.

[6] C. Chen, Z. Hu, Y. Wang, B. Wei, and Q. Xia, "New edge detection method for images of infrared thermal imager", Infrared Laser Eng., vol. 43, pp. 319-322, January 2014.

[7] H. Jiang, W. Yuan, X. Zhang, X. Zhang and W. Li, "Application of wavelet transformation in the process of abdominal MRI", Control Eng., vol. 10, pp. 360-363, April 2003.

[8] R. C. Gonzalez, R. E. Woods and S. L. Eddins, "Digital Image Processing (MATLAB Version)". Beijing: Publishing House of Electronics Industry, 2007.

[9] W. Zhang "Image denoising algorithm of refuge chamber by combining wavelet transform and bilateral filtering", Int. J. Mining Sci. Technol., vol. 2, pp. 221-224, June 2013.

[10] C. Wang, B. Ye, X. Huang and C. Huang, "Research of the detection system of external diameter of bearings based on computer vision”, Microcomput. Inform., vol. 2 , pp. 205-207, May 2006.

[11] E. P. Lyers, O. R. Mitchell, M. L. Akey, and A. P. Reeves, "Subpixel measurements using a moment-based edge operator", IEEE Trans. Pattern Anal. Machine Intell., vol. 11, no. 12, pp. 12931307, Dec. 1989

[12] K. Jia and J. Xue "Research of gait recognition technology based on Zernike moments and bp network", J. Northwestern Polytech. Univ., vol. 28. pp. 669-673., May 2010.

[13] W. Zhang, D. Li, and F. Ye, "A subpixel edge detection method based on sigmoid function fitting", J. South China Univ. Technol. (Nat. Sci. Ed.), vol. 37, pp. 40-43, October 2009.

[14] S. Ghosal and R. Mehrotra. "Orthogonal moment operators for subpixel edge detection", Pattern Recognit., vol. 26, pp. 295-306, February 1993

[15] G. A. Papakostas, Y. S. Boutaus, C. N. Papaodysseus, and D. K. Fragoulis, "Numedcal error analysis in Zernike moments computa- 
tion", Image Comput. Vision, vol. 24, pp. 960-969, September 2006.

[16] J. Cui and J. Tan "A subpixel edge positioning algorithm based on Zernike moments", Opt. Tech., vol. 31, pp. 779-782, May 2005.
[17] Y. Qu, R. Li and Y. Bai, R. Li, and G. Ma, "High-speed Zernike moment edge operator with the model of $9 \times 9$ ", Photoelect. Laser, vol. 21, pp. 1683-1687, Nov. 2010.

Received: September 16, 2014

(C) Zhang Weipeng; Licensee Bentham Open.

This is an open access article licensed under the terms of the Creative Commons Attribution Non-Commercial License (http://creativecommons.org/licenses/ by-nc/4.0/) which permits unrestricted, non-commercial use, distribution and reproduction in any medium, provided the work is properly cited. 